\title{
Effect of the Illuminating Point Spread Function and Roughness on the Speckle Correlation Field
}

\author{
Mohamed Mansour El-Nicklawy ${ }^{1}$, Amin Fahim Hassan'1, Adel Ebrahim Elmahdy ${ }^{*}$, \\ Afaf Mahmoud Abd-Rabou1, Ezzulddin Abdoulsahib Eesee ${ }^{1,2}$ \\ ${ }^{1}$ Department of Physics, Faculty of Science, Helwan University, Cairo, Egypt \\ ${ }^{2}$ Department of Science, Open Educational College, Baghdad, Iraq \\ Email: *adel_elmahdy@science.helwan.edu.eg
}

How to cite this paper: El-Nicklawy, M.M., Hassan, A.F., Elmahdy, A.E., Abd-Rabou, A.M. and Eesee, E.A. (2019) Effect of the Illuminating Point Spread Function and Roughness on the Speckle Correlation Field. Optics and Photonics Journal, 9, 165-177.

https://doi.org/10.4236/opj.2019.911015

Received: September 26, 2019

Accepted: November 1, 2019

Published: November 4, 2019

Copyright $\odot 2019$ by author(s) and Scientific Research Publishing Inc. This work is licensed under the Creative Commons Attribution International License (CC BY 4.0).

http://creativecommons.org/licenses/by/4.0/

\begin{abstract}
Under conditions differing from those subjected for central limit theorem, the spatial autocorrelation function of speckle pattern resulting from illuminated rough surface is investigated. Its dependence on different illuminating apertures and the average of the roughness heights is presented theoretically and experimentally. The experiments were carried out using a set of circular and square apertures having different sizes. The results indicate that, increasing the size of the illuminating aperture leads to a decrease in the width of the main lobe of the spatial autocorrelation function.
\end{abstract}

\section{Keywords}

Speckle Pattern, Autocorrelation Function, Rough Surface

\section{Introduction}

As a result of illuminating a rough surface with coherent or partially coherent light, interference between reflected or transmitted waves occurs. Due to the random roughness heights of the surface, the interfering beams are of random phases. This leads to intensities of random values with random spatial distribution called speckle patterns [1] [2] [3]. The statistical properties of laser speckle patterns are of great importance in speckle metrology techniques. These techniques are used for solving numerous problems in industry, for example strain measurement, velocity measurement and surface roughness measurement. The speckle correlation is the most important statistical property of the speckle pattern. It gives important information about the surface quality of materials and the spatial structure of the surface heights [4] [5] [6] [7]. Many researchers in- 
vestigated the statistical optical properties of fully developed speckle pattern. They considered the random phases of the interfering waves are uniformly distributed in the range from $-\pi$ to $+\pi$ and the number of the interfering waves contributing to any point of the observation plane is large enough [1] [8] [9] [10] [11] [12]. N. Yu. Mysina et al. [13] have investigated the optical statistical properties of the speckle fields at two points in the Fraunhofer diffraction region with four different shapes of the scatters apertures. The apertures were square, annular square, triangle and annular triangle. They have shown that the region of the field correlation has considerably larger limits when using annular shape specially the square one. Uno et al. [14] have observed clustering or snake-like appearance when using a ring-slit illuminating a rough surface. In addition, long and ringing tails of the intensity correlation function of the speckle pattern have been observed. Asakura et al. [15] have investigated the intensity correlation of the speckle patterns produced by different structure apertures. They were circular, ring-slit, half segment, two-quarter segments, three-sixth segments and four-eighth segments apertures. The results have shown that the spatial distribution of the speckle and its directionality were affected by the structure of the used aperture. Maack et al. [16] have investigated the effect of a reference beam on the spatial frequency spectrum of the speckle field when a rough object is illuminated with a circular aperture. They have found that the ratio of the speckle and the reference intensities control the accuracy of the measurement of the experimental setup.

The majority of the publications in this respect rely on the central limit theorem which considers limited conditions on the statistics of the roughness. Under such conditions, the speckle correlation and contrast do not depend on the statistical characteristics of the roughness heights. The present work deals with studying the theoretical and experimental dependence of the statistical properties of laser speckle mainly the autocorrelation function of the speckle fields on the aperture illuminating shape and its dimension as well as the roughness root mean square beyond the conditions of the central limit theorem.

\section{Theoretical Background}

\subsection{Correlation of the Speckle Field}

The autocorrelation function between two intensities of speckle fields located at positions $r_{1}$ and $r_{2}$ on the observation plane is given from the ensemble average of $I\left(r_{1}\right)$ and $I\left(r_{2}\right)$ as [1]

$$
R_{I}\left(r_{1}, r_{2}\right)=\left\langle I\left(r_{1}\right) I\left(r_{2}\right)\right\rangle,
$$

where $\langle\cdots\rangle$ denotes the ensemble average.

Under the statistical conditions of the central limit theorem, the autocorrelation function according to Goodman [1] can be written as

$$
R_{I}\left(r_{1}, r_{2}\right)=\left\langle I\left(r_{1}\right) I\left(r_{2}\right)\right\rangle=\left\langle I_{s}\right\rangle^{2}+\left|\left\langle A\left(r_{1}\right) A^{*}\left(r_{2}\right)\right\rangle\right|^{2} .
$$

where $A\left(r_{1}\right)$ and $A\left(r_{2}\right)$ are the complex field amplitudes at the points $r_{1}$ and 
$r_{2}$, respectively. The asterisk denotes the conjugate complex. Considering

$$
\left\langle I\left(r_{1}\right)\right\rangle=\left\langle I\left(r_{2}\right)\right\rangle=\left\langle I_{s}\right\rangle \text { and } \mu_{A}\left(r_{1}, r_{2}\right)=\frac{\left\langle A\left(r_{1}\right) A^{*}\left(r_{2}\right)\right\rangle}{\left\langle I_{s}\right\rangle},
$$

where $\mu_{A}\left(r_{1}, r_{2}\right)$ is the complex coherence factor of the speckle field, then Equation (2) can be written as [1] [16]

$$
R_{I}\left(r_{1}, r_{2}\right)=\left\langle I_{s}\right\rangle^{2}\left(1+\left|\mu_{A}\left(r_{1}, r_{2}\right)\right|^{2}\right) .
$$

In fact $\mu_{A}$ is the Fourier transform of the intensity distribution of the object and $\left\langle I_{s}\right\rangle$ is the average intensity of the speckle pattern.

It is worth noting that $\left\langle I_{s}\right\rangle$ in Equation (4) obeys according to Goodman [1], the central limit theorem.

Maack et al. [16] restricted his study of speckle correlation on the effect of the illuminating point spread function. It is by considering $\left\langle I_{s}\right\rangle$ is a constant number does not depend on the statistical characteristics of the rough surface.

To achieve the aim of the present work, the dependence of $R_{I}$ on the root mean square of the roughness heights, we have to consider a formula for $\left\langle I_{s}\right\rangle$ depending on such statistical parameters. According to [3], $\left\langle I_{s}\right\rangle$ is given by

$$
\begin{gathered}
\left\langle I_{s}\right\rangle=N+N(N-1)\left(x^{2}+y^{2}\right) \gamma, \\
x=\langle\cos (\varphi)\rangle=\int_{-\infty}^{\infty} \cos (\varphi) P(\varphi) \mathrm{d} \varphi=\exp \left(-\sigma_{\varphi}^{2} / 2\right), \\
\text { and } y=\langle\sin (\varphi)\rangle=\int_{-\infty}^{\infty} \sin (\varphi) P(\varphi) \mathrm{d} \varphi=0 .
\end{gathered}
$$

where $N$ is the number of the interfering beams and $\gamma$ is the mutual degree of the temporal coherence between the interfering beams. $\gamma$ tends to one when using monochromatic light. $P(\varphi)$ is a Gaussian phase probability density distribution with zero mean value and $\sigma_{\varphi}$ is the root mean square of the random phase $(\varphi)$. It is to be noted that, the statistical conditions of formula (5) do not obey the limited statistical conditions of the central limit theorem. For formula (4) to be still valid, formula (5) of $\left\langle I_{s}\right\rangle$ is modified such that it verifies as nearly as possible the statistical conditions of the central limit theorem. It follows:

1) $\left\langle I_{s}\right\rangle$ is normalized through dividing it by $N^{2}$.

2) let $N$ tends to infinity which is one of the conditions of the central limit theorem.

3) consider $\sigma_{\varphi}$ to be of large values which is a second condition of the central limit theorem.

Noting that a Gaussian distribution of large $\sigma_{\varphi}$ approaches to a uniform distribution which is one of the limited conditions. Under such modification we get:

$$
\left\langle I_{s}\right\rangle=\exp \left(-\sigma_{\varphi}^{2}\right) .
$$

The speckle contrast reaches one as $\sigma_{\varphi}$ takes large values and thus the central limit theorem is reached [3]. As a consequence of the obtained formula of 
$\left\langle I_{s}\right\rangle, R_{I}\left(r_{1}, r_{2}\right)$ is given by

$$
R_{I}\left(r_{1}, r_{2}\right)=\exp \left(-2 \sigma_{\varphi}^{2}\right)\left(1+\left|\mu_{A}\left(r_{1}, r_{2}\right)\right|^{2}\right)
$$

Equation (6) holds only for large values of $\sigma_{\varphi}$. It indicates that, the correlation of the speckle field decreases with increasing $\sigma_{\varphi}$.

\subsection{Circular and Square Illuminating Apertures}

To investigate the effect of the illuminating point spread function, circular and square apertures of various dimensions are chosen. The correlation $\left(R_{I}\right)$ of the speckle field produced when using a circular aperture is given by [14]

$$
R_{I}\left(r_{1}, r_{2}\right)=\left\langle I_{s}\right\rangle^{2}\left(1+\left|\frac{2 J_{1}(\mathrm{kar} / f)}{\mathrm{kar} / f}\right|^{2}\right) .
$$

where $r=\sqrt{x^{2}+y^{2}}$ and $a$ is the radius of the aperture, and $J_{1}$ is the Bessel function of the first kind of order one.

The correlation at the speckle field produced when using a square aperture is given by [13]

$$
R_{I}\left(r_{1}, r_{2}\right)=\left\langle I_{s}\right\rangle^{2}\left(1+\left(\frac{\sin \alpha}{\alpha}\right)^{2}\left(\frac{\sin \beta}{\beta}\right)^{2}\right)
$$

where $\alpha=\frac{k L x}{2 f}$ and $\beta=\frac{k L y}{2 f}, L$ is the side length of the square aperture.

$$
\left|\frac{2 J_{1}(\mathrm{kar} / \mathrm{f})}{\mathrm{kar} / \mathrm{f}}\right|^{2} \text { and }\left(\frac{\sin \alpha}{\alpha}\right)^{2}\left(\frac{\sin \beta}{\beta}\right)^{2} \text { arise from the diffraction phenomena }
$$

of a circular and square apertures, respectively. They are called the intensity point spread functions of the illuminating apertures.

Figures 1(a)-(d) and Figures 2(a)-(d) show the behavior of the speckle correlation with the speckle separated distance $\Delta x$ for circular and square illuminating apertures, respectively. The figures are evaluated numerically, for different values of $\sigma_{\varphi}$, through the theoretical formula given by Equation (6). The figures indicate that the speckle correlation function decreases with increasing both of $\Delta x$ and $\sigma_{\varphi}$ which is directly proportional to the square root of the average of the square heights of the roughness. This is due to the fact that by increasing $\sigma_{\varphi}$ the phase randomization of the scattered interfering waves increases leading to a decrease in the spatial autocorrelation function of the speckle. Figures 3(a)-(d) show the decaying of the speckle correlation at arbitrary point $x=33 \mu \mathrm{m}$ on the speckle pattern plane, with increasing $\sigma_{\varphi}$ for circular and square illuminating apertures, respectively.

\section{Experimental Work}

\section{Experimental Setup}

Schematic diagram of the optical experimental setup used to generate speckle patterns is shown in Figure 4. 


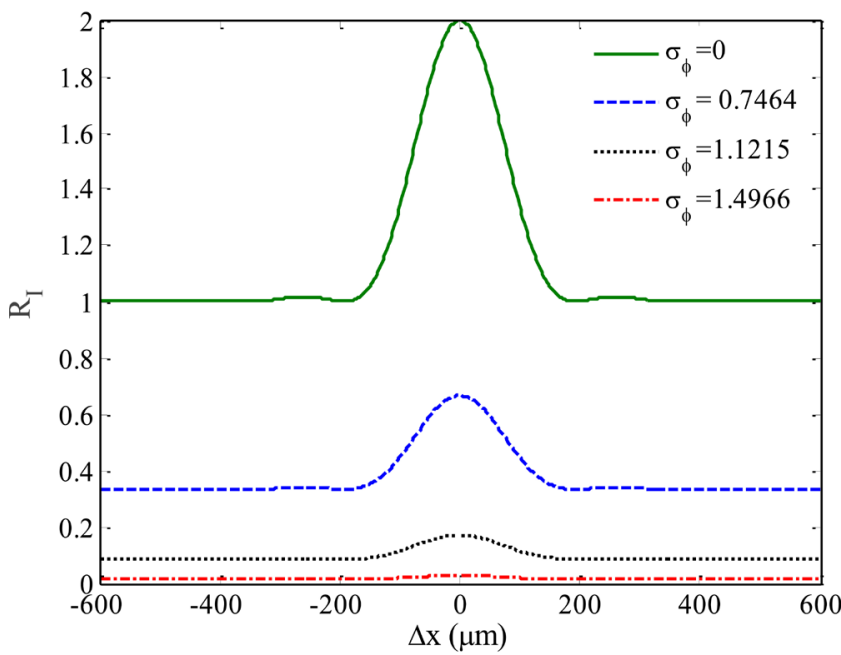

(a)

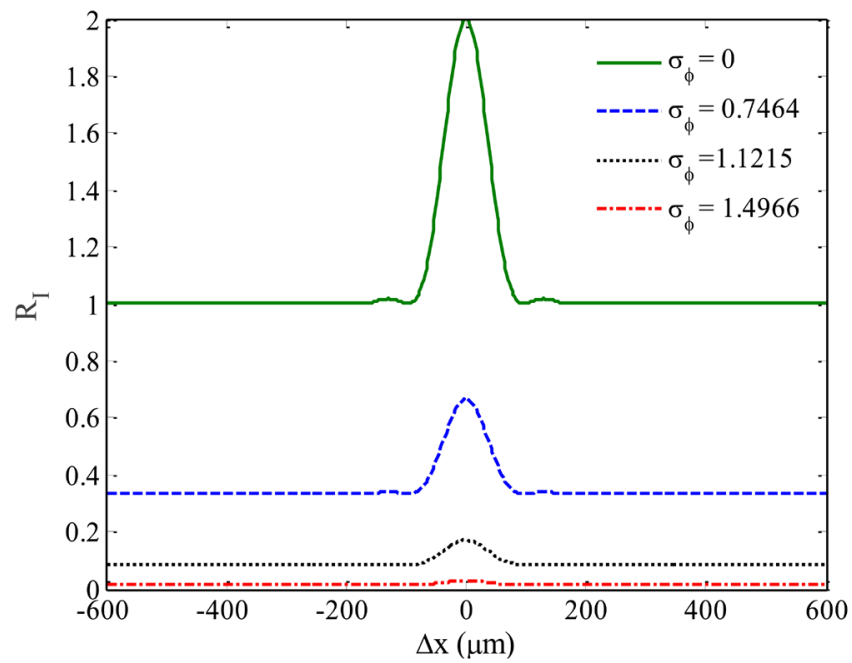

(c)

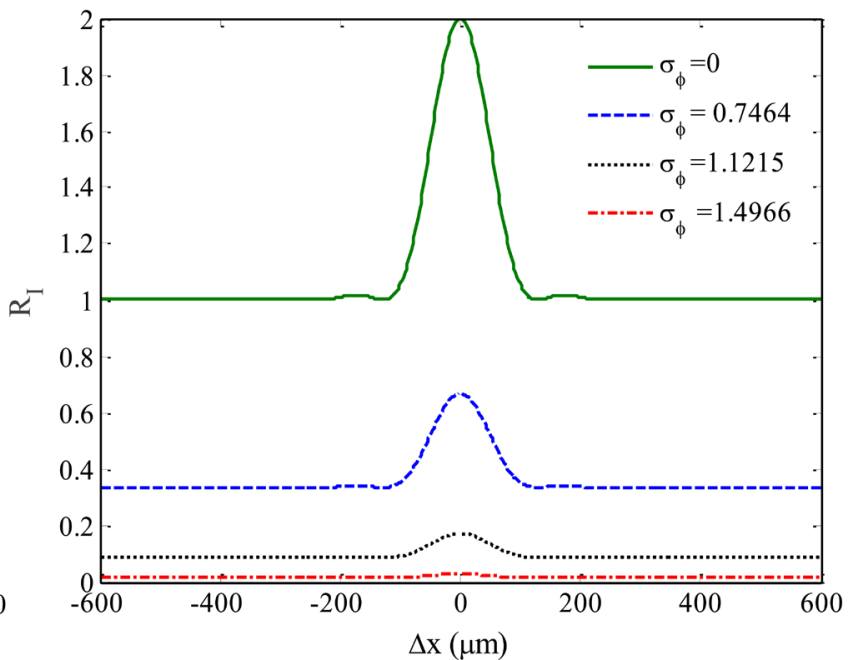

(b)

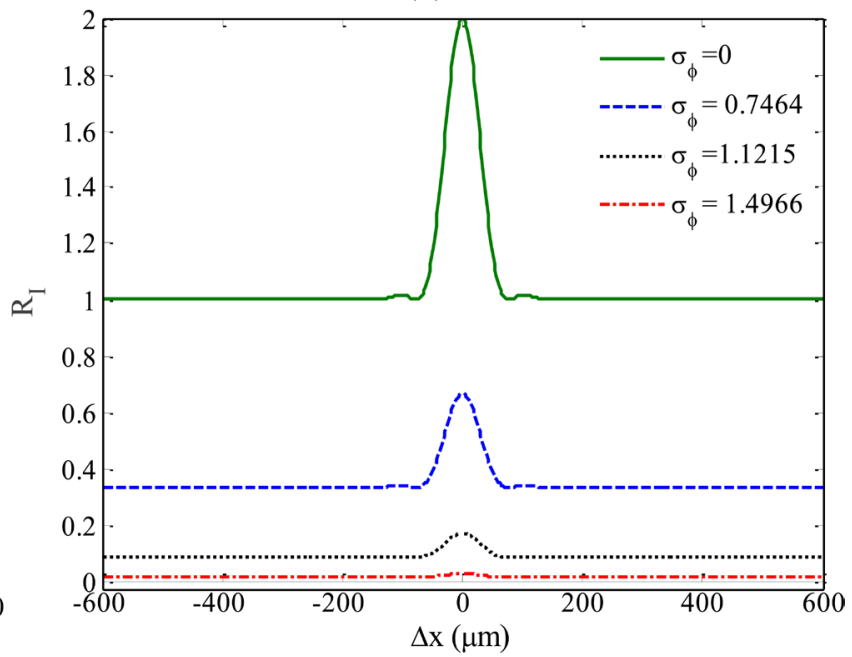

(d)

Figure 1. The dependence of the autocorrelation function on the separation $\Delta x$ computed for a circular aperture of (a) $D=0.4$ $\mathrm{mm}$, (b) $D=0.6 \mathrm{~mm}$, (c) $D=0.8 \mathrm{~mm}$ and (d) $D=1 \mathrm{~mm}$.

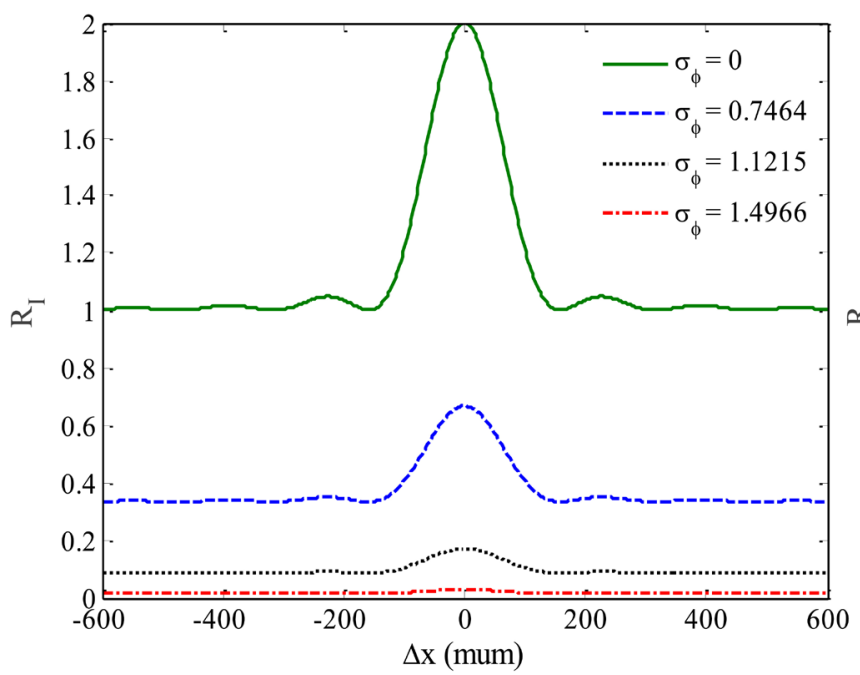

(a)

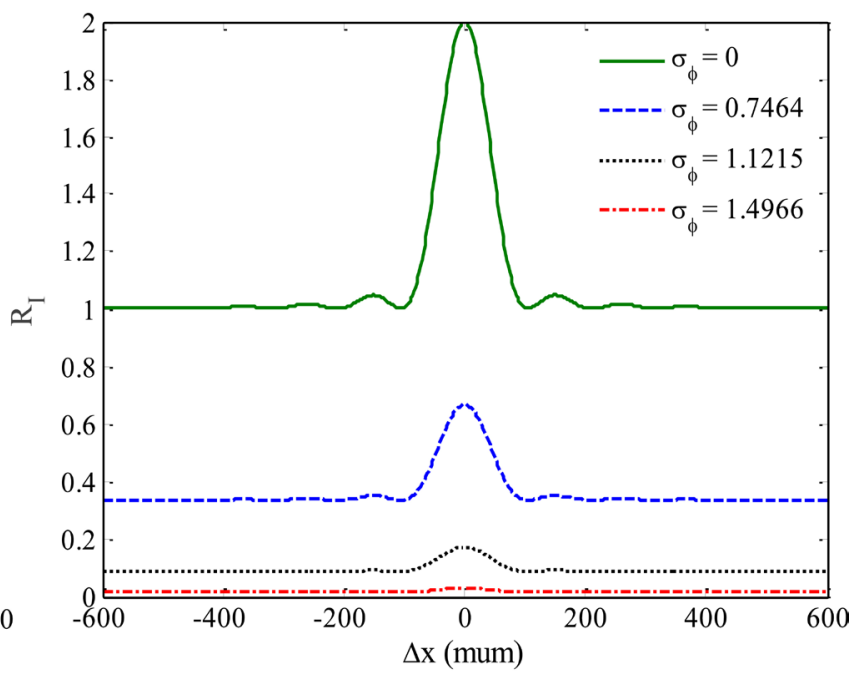

(b) 


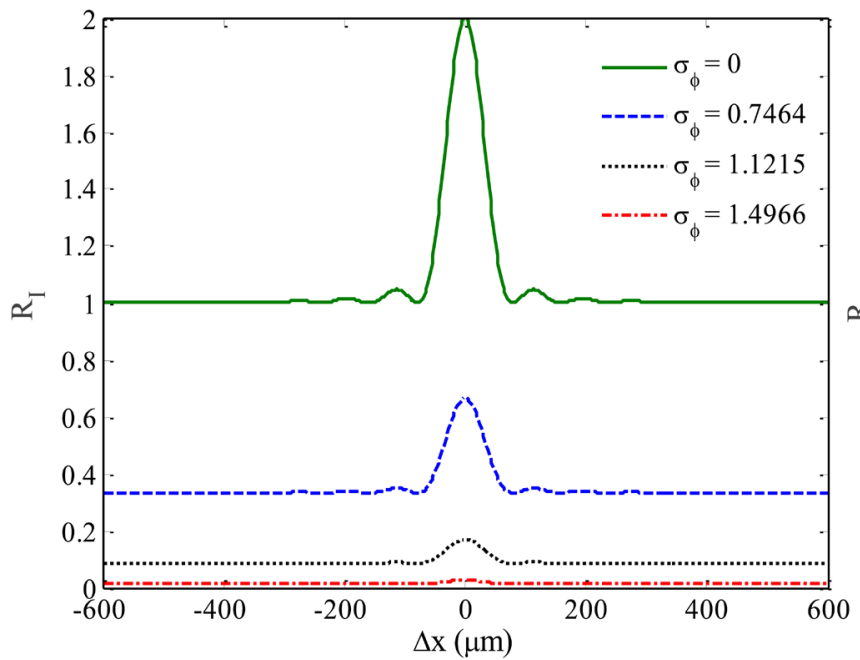

(c)

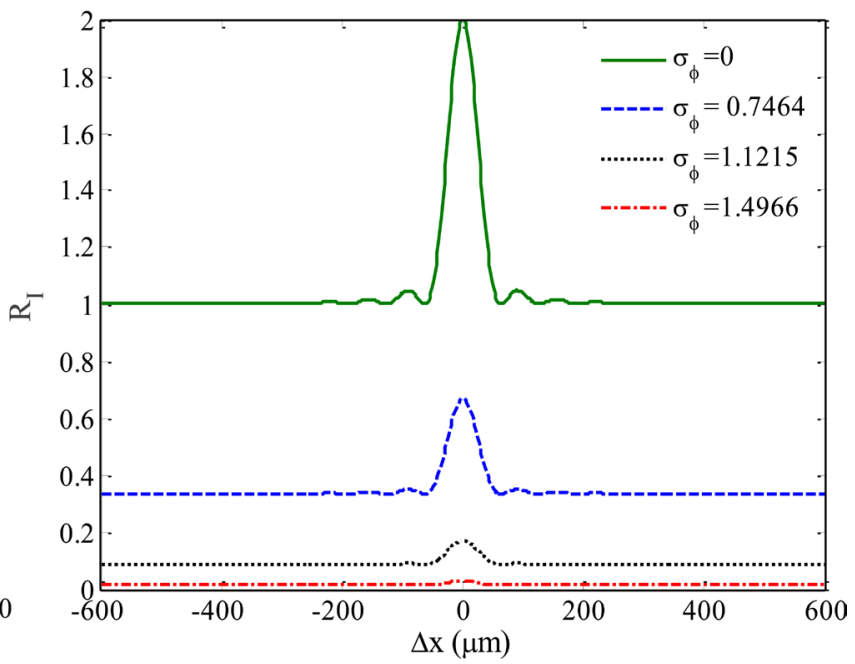

(d)

Figure 2. The dependence of the autocorrelation function on the separation $\Delta x$ computed for a square aperture of (a) $L=0.4 \mathrm{~mm}$, (b) $L=0.6 \mathrm{~mm}$, (c) $L=0.8 \mathrm{~mm}$ and (d) $L=1 \mathrm{~mm}$.

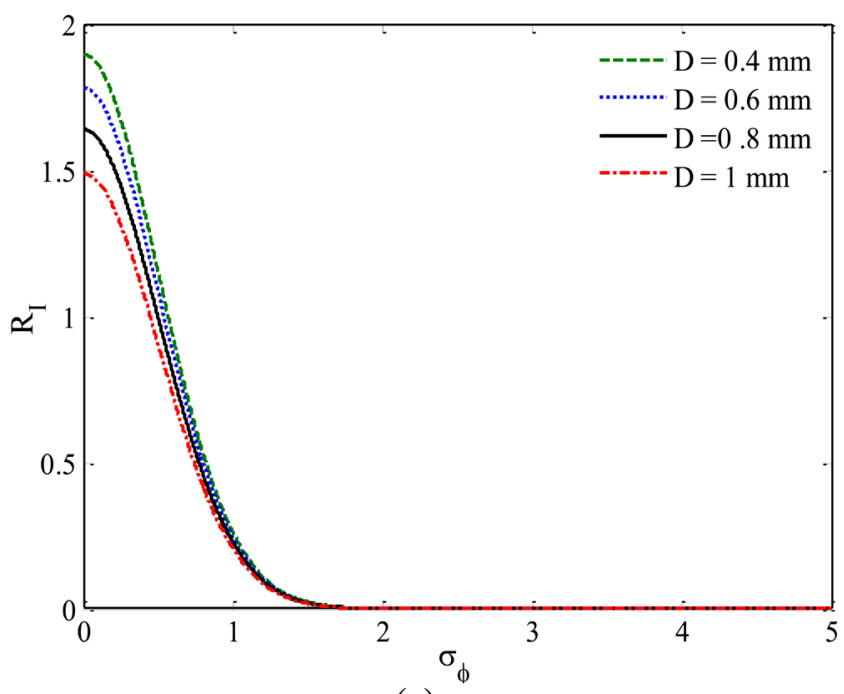

(a)

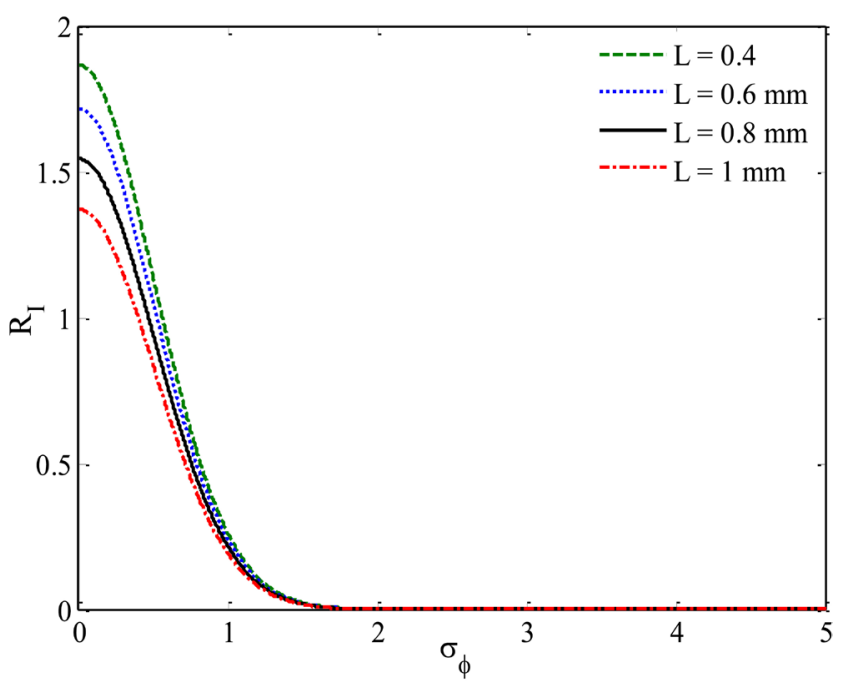

(b)

Figure 3. The dependence of the autocorrelation function on $\sigma_{\varphi}$ computed for (a) circular aperture and (b) square aperture having different dimensions.

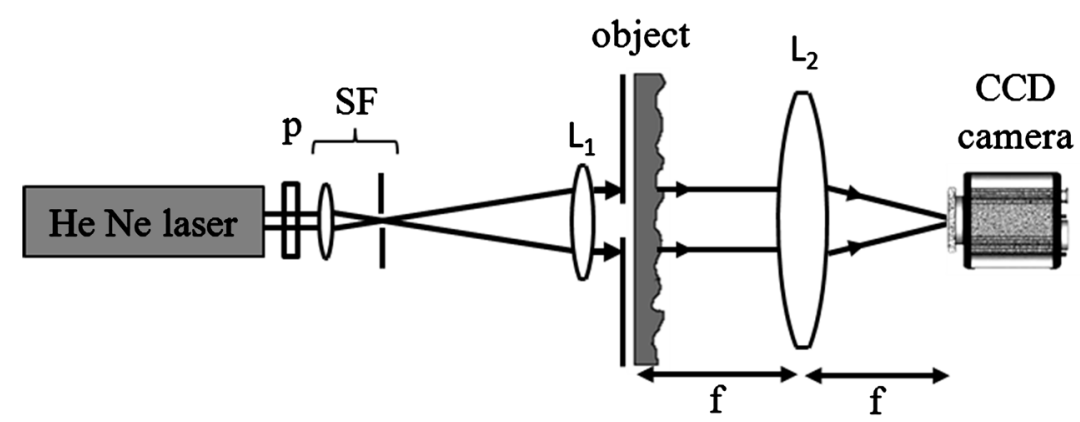

Figure 4. Experimental setup for speckle formation and recording: $P$, polarizer; SF, spatial filter; object is consisting of an aperture and a transmission diffuser; $L_{1}$ and $L_{2}$, two lenses. 
A He-Ne laser was expanded and collimated by the use of a spatial filter (SF) and a lens $\left(L_{1}\right)$. The object consists of a combination of an aperture and different transparent diffusers. Circular and square apertures having different dimensions are used. To obtain an optical Fourier transform, the object has to be located at the front focal plane of the imaging lens $\left(L_{2}\right)$ and the CCD Camera is located at the back focal plane of $\left(L_{2}\right)$. Speckle patterns are recorded on the CCD Camera $(1920 \times 2560$ pixels, $2.2 \mu \mathrm{m}$ pixel size, 8 bit $)$.

\section{Results}

With the aid of the optical setup shown in Figure 4, the speckle interferograms are recorded for different types and dimensions of the illuminating apertures. The autocorrelation function of the field is numerically evaluated according to Winner-Kinchine theory [14] by applying the Fourier transform twice on the speckle recorded intensity.

Figure 5(a) \& Figure 5(b) represent the speckle patterns for a circular illuminating aperture of diameter $0.4 \mathrm{~mm}$ for transparent rough surface of average roughness heights $1.98 \mu \mathrm{m}$ and $7.6 \mu \mathrm{m}$, respectively. While Figure 6(a) \& Figure 6(b) represent the speckle patterns for illuminating aperture of diameter $1 \mathrm{~mm}$ for the same rough surfaces.

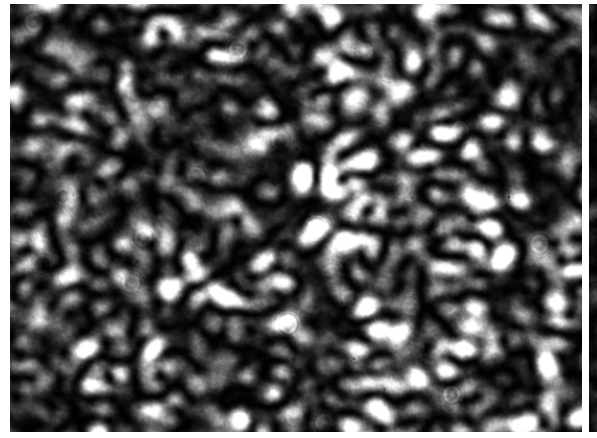

(a)

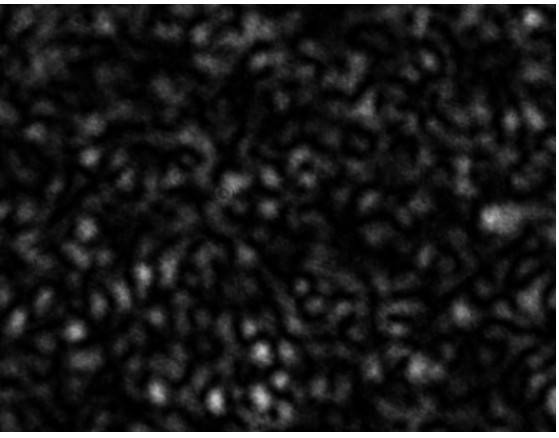

(b)

Figure 5. Speckle patterns for circular aperture of diameter $0.4 \mathrm{~mm}$ and roughness; (a) $R_{a}$ $=1.98 \mu \mathrm{m}$ and (b) $R_{a}=7.6 \mu \mathrm{m}$.

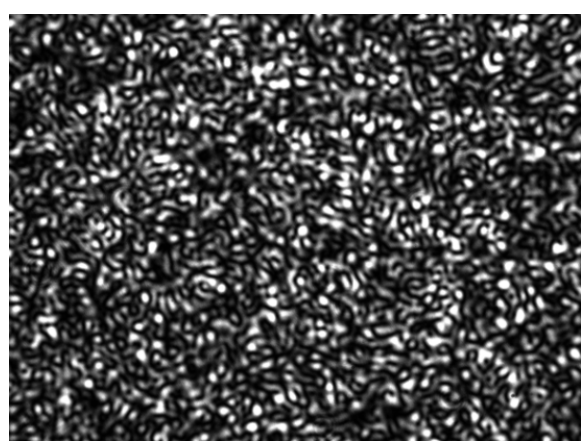

(a)

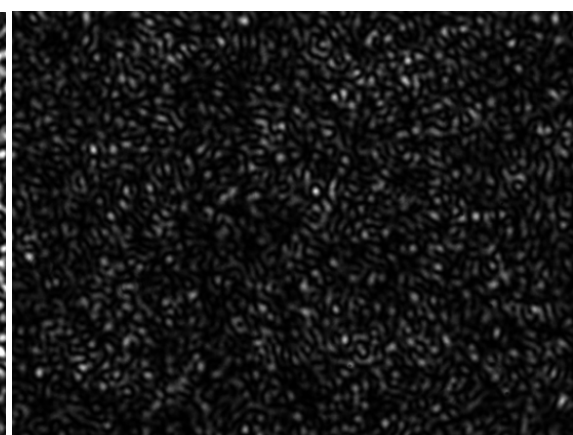

(b)

Figure 6. Speckle patterns for circular aperture of diameter $1 \mathrm{~mm}$ and roughness; (a) $R_{a}=$ $1.98 \mu \mathrm{m}$ and (b) $R_{a}=7.6 \mu \mathrm{m}$. 
Figure 7(a) \& Figure 7(b) and Figure 8(a) \& Figure 8(b) show the speckle patterns for a square aperture of 0.4 and $1 \mathrm{~mm}$ side lengths, respectively.

Figures 9(a)-(d) and Figures 10(a)-(d) represent a section profile of the normalized autocorrelation function maps that numerically evaluated through the Fourier transform of the recorded intensities of the speckle patterns when using circular and square apertures, respectively. Each figure is for particular roughness with the dimension of the illuminating aperture as a parameter. The considered average roughness heights are $1.98 \mu \mathrm{m}, 3.8 \mu \mathrm{m}, 5 \mu \mathrm{m}$ and $7.6 \mu \mathrm{m}$ and the apertures dimensions are $0.4 \mathrm{~mm}, 0.6 \mathrm{~mm}, 0.8 \mathrm{~mm}$ and $1 \mathrm{~mm}$.

The figures show as expected that the speckle size decrease as the dimension of the aperture increases.

The apparent crowding of the $R_{I}$ curves as the aperture tends to $1 \mathrm{~mm}$ is due to the nonlinear behavior of the Bessel function as well as the Sinc function. Moreover, it is observed that the crowding density increases with increasing the roughness by keeping the dimension of the aperture unchanged. This behavior is due to the relatively greater aperture area that leads to shrinking the illuminating point spread function and thus to $R_{I}$. This behavior is more pronounced as the square aperture is considered because of the greater area of the square aperture in comparison with the circular aperture of the same dimension.

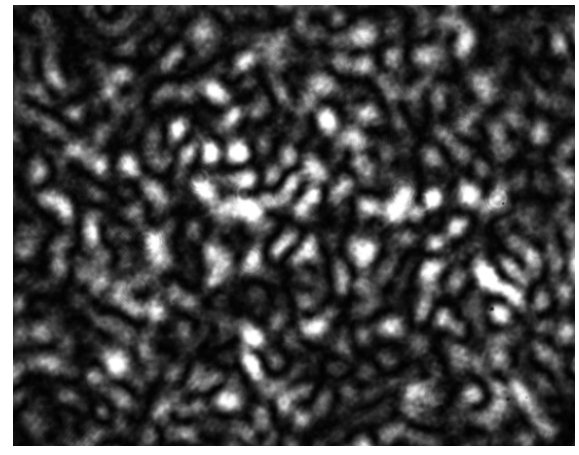

(a)

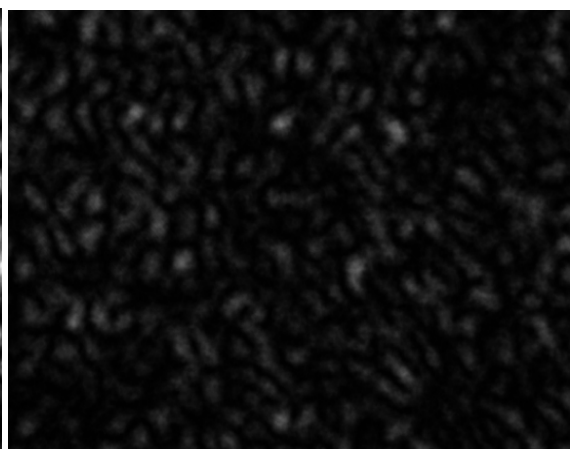

(b)

Figure 7. Speckle patterns for square aperture of side length $0.4 \mathrm{~mm}$ and roughness; (a) $R_{\mathrm{a}}=1.98 \mu \mathrm{m}$ and (b) $R_{\mathrm{a}}=7.6 \mu \mathrm{m}$.

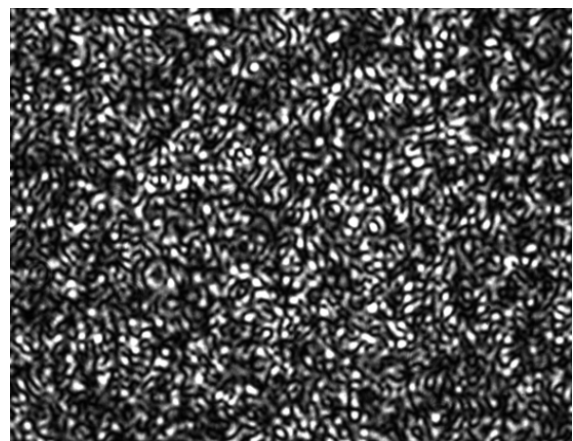

(a)

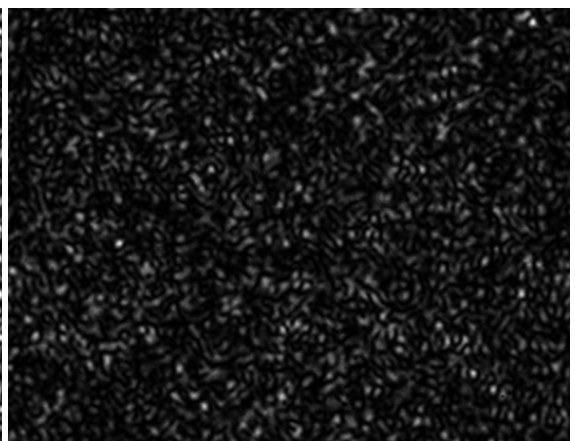

(b)

Figure 8. Speckle patterns for square aperture of side length $1 \mathrm{~mm}$ and roughness; (a) $R_{a}$ $=1.98 \mu \mathrm{m}$ and (b) $R_{a}=7.6 \mu \mathrm{m}$. 


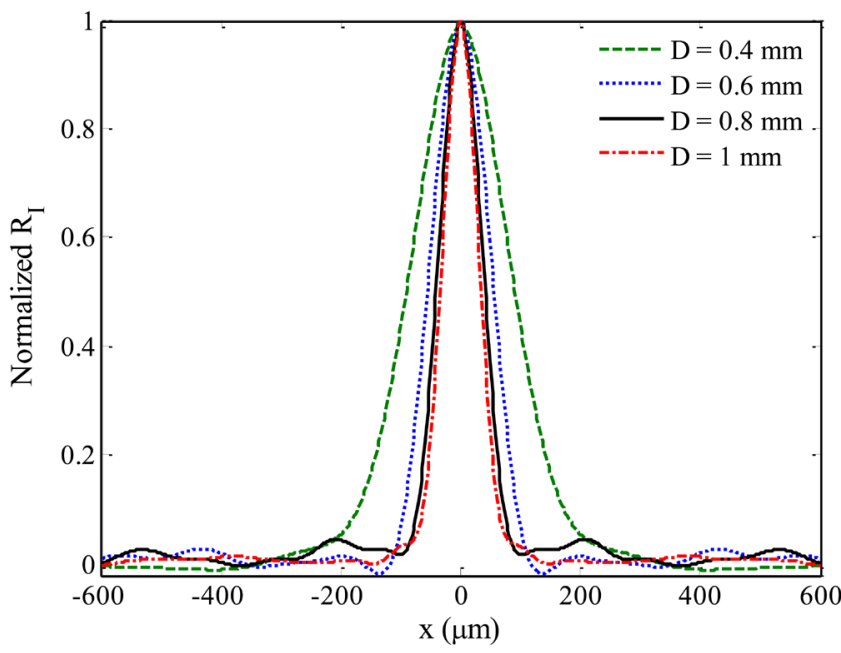

(a)

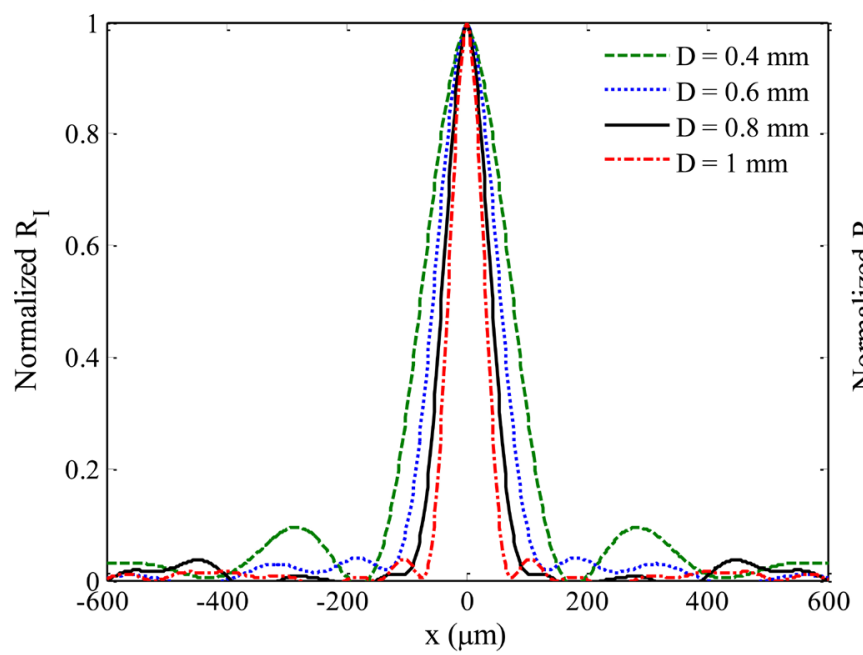

(c)

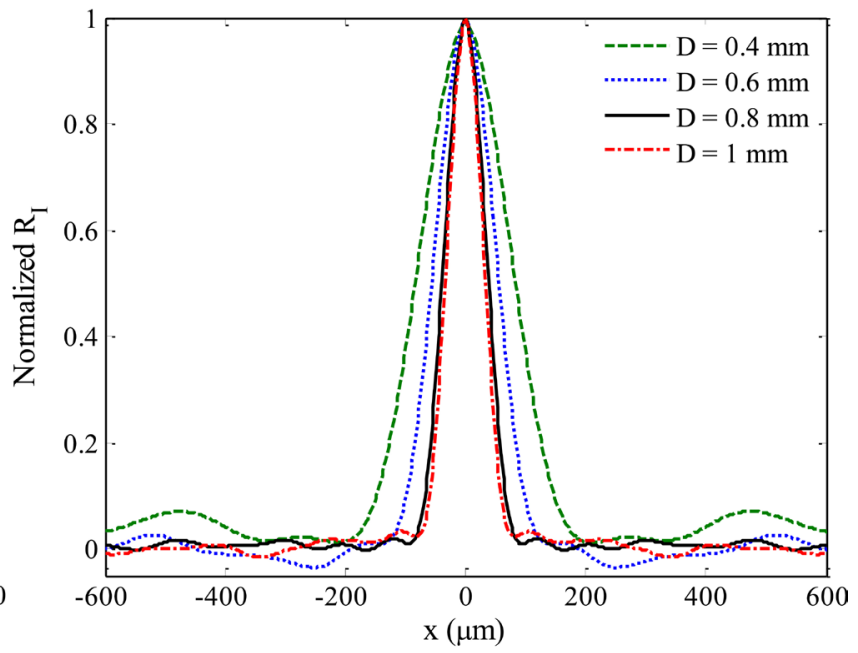

(b)

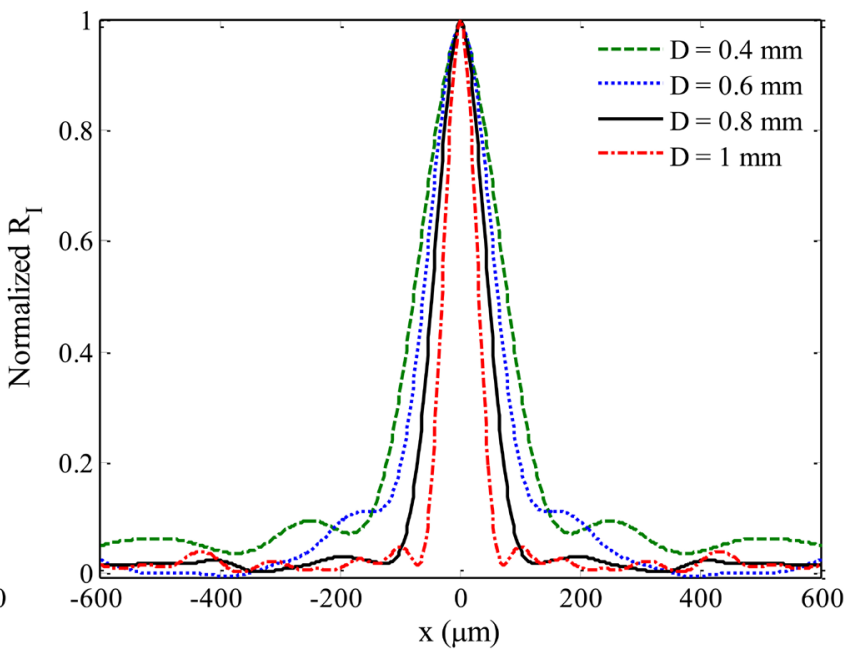

(d)

Figure 9. The normalized autocorrelation functions of the experimentally recorded speckle patterns when the rough surface masked with a circular aperture with different diameters for surfaces of average roughness $R_{a}$ : (a) $1.98 \mu \mathrm{m},(\mathrm{b}) 3.8 \mu \mathrm{m},(\mathrm{c}) 5 \mu \mathrm{m}$, (d) $7.6 \mu \mathrm{m}$.

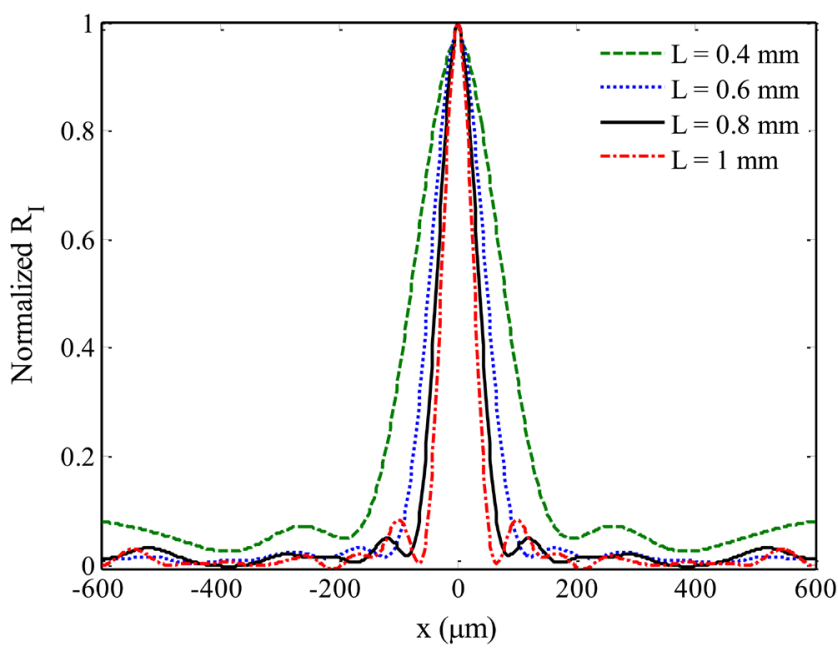

(a)

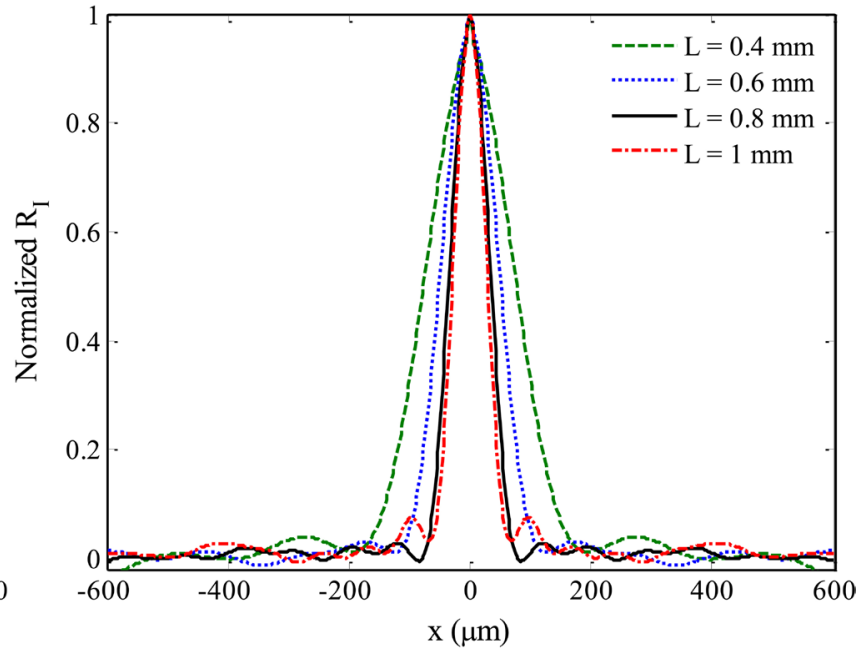

(b) 


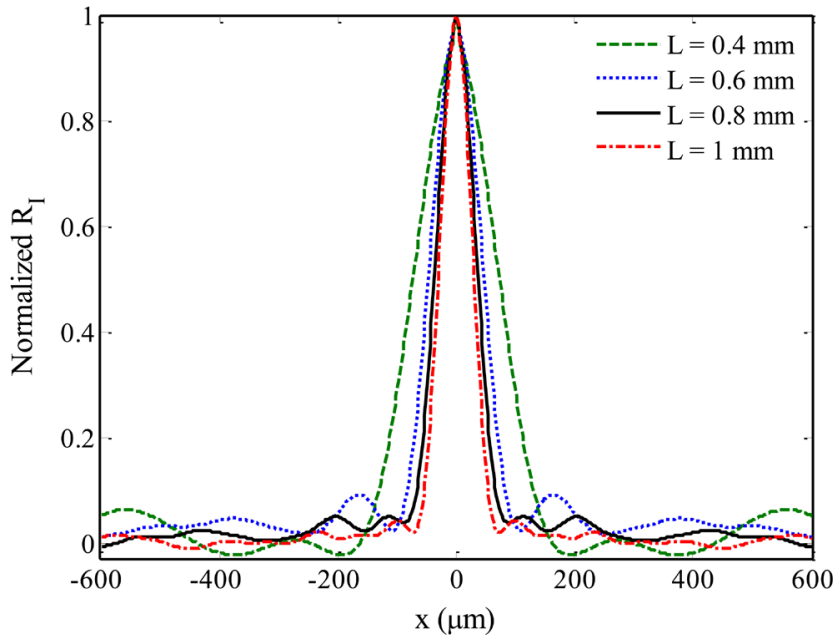

(c)

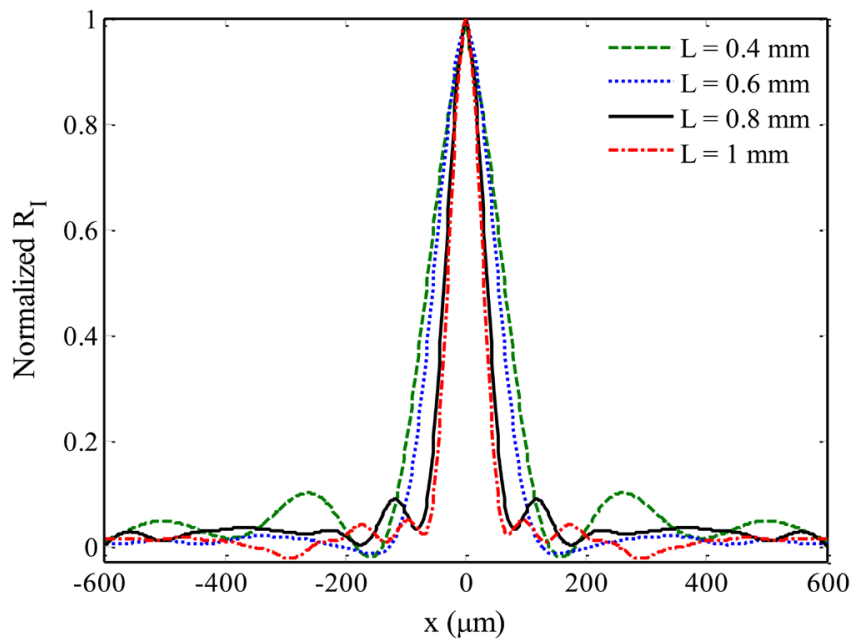

(d)

Figure 10. The normalized autocorrelation functions of the experimentally recorded speckle patterns when the rough surface masked with a square aperture with different dimensions for surfaces of average roughness $R_{a}$ : (a) $1.98 \mu \mathrm{m}$, (b) $3.8 \mu \mathrm{m}$, (c) $5 \mu \mathrm{m}$, (d) $7.6 \mu \mathrm{m}$.

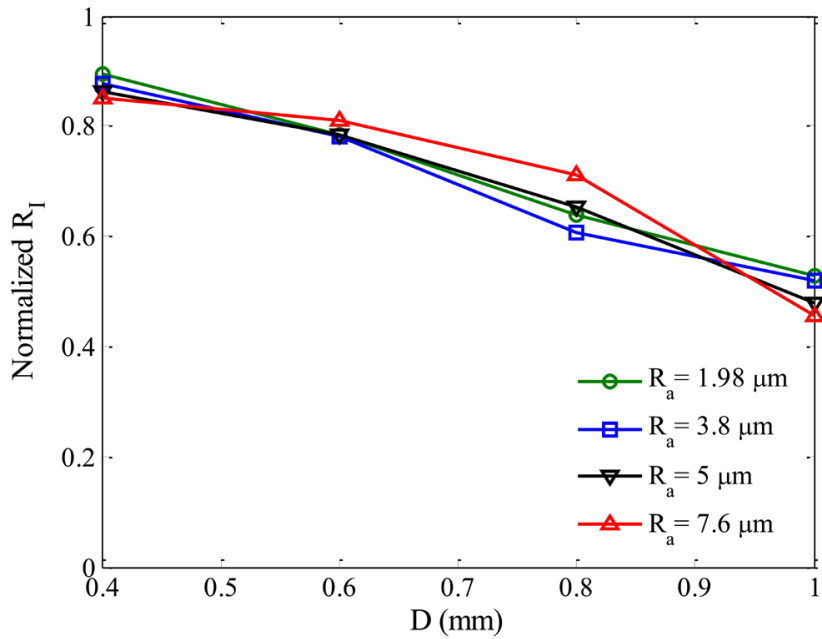

(a)

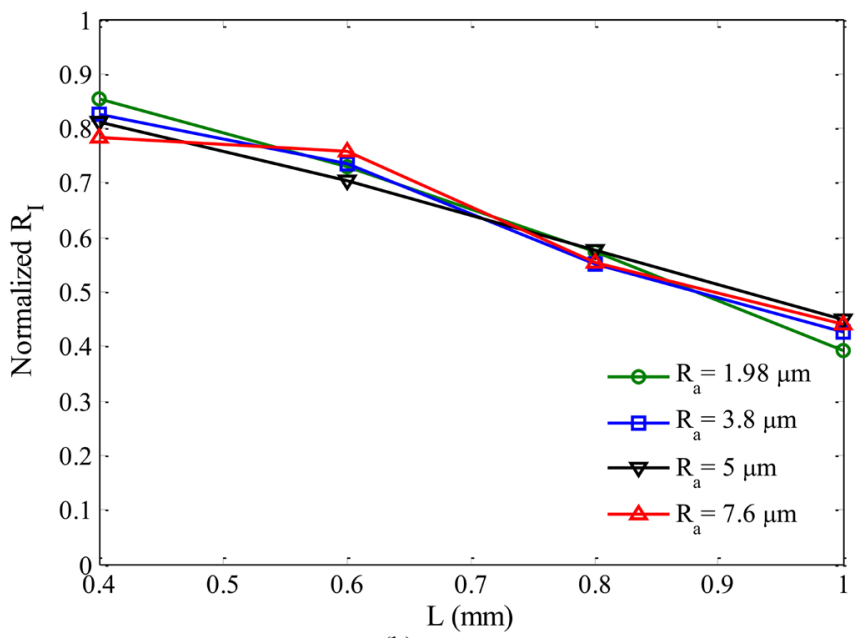

(b)

Figure 11. The normalized autocorrelation of speckles as a function of (a) the circular aperture diameter $D$ and (b) the square aperture side length $L$ for a fixed separation distance $x=33 \mu \mathrm{m}$ for different $R_{a}$.

As a result, the behavior of the speckle correlation function obtained experimentally agrees with that numerically obtained through the theoretical formula given by Equation (6). An important general result indicated theoretically and experimentally is the speckle correlation decreases with increasing the speckle spacing $(x)$.

To have a general overview of the parameters affecting the normalized autocorrelation functions, Figure 11(a) \& Figure 11(b) are drawing to illustrate the dependence of the normalized autocorrelation functions on the aperture size for circular and square apertures, respectively. The values of this function have been extracted from the spatial correlation distributions, Figure 9 and Figure 10, at a fixed distance $x=33 \mu \mathrm{m}$ with $R_{a}$ as a parameter. The figures show that the nor- 
malized autocorrelation function decreases with increasing the aperture sizes and is practically independent of the surface roughness $R_{a}$.

\section{Discussion}

Speckle pattern is a result of two optical phenomena namely interference between scattered waves acquired random phases from a rough surface and diffraction due to the illuminating aperture. Thus the speckle intensity arises from spatial intensity of interference pattern modulated by spatial intensity of diffraction pattern.

The correlation between the speckle intensities is basically attributed to those two optical phenomena. The characteristics of the produced random spatial interference pattern are referred to the statistical physical conditions of the rough surface, while the modulated factor of the diffraction is attributed to the conditions of the imaging system including the shape and dimensions of the illuminating aperture. These physical bases of arising speckle correlation lead us to consider the speckle correlation function as a convolution of the two spatial intensity distributions of interference and diffraction phenomena. The effect of one of them on the behavior of the speckle correlation function can overcome the effect of the second one depending on the following factors:

1) The phase probability density distribution which is acquired by the scattering waves.

2) The phase correlation function of the roughness and its correlation length.

3) The shape and dimensions of the illuminating aperture and in turn the resulting illuminating point spread function of the imaging system.

In general, the correlation between the speckle patterns mainly arises from the correlation between the interfering waves which causing the speckle pattern.

\section{Conclusion}

In conclusion, we have presented the theoretical and experimental dependence of the spatial autocorrelation function of the speckle intensity on the aperture shape, the aperture size and the roughness of transparent diffusers. A significant effect of the aperture size on the spatial normalized autocorrelation function is observed considering both the circular and square apertures. Increasing the size of the illuminating aperture permits more number of waves to be incident on the scattering surface and in turn more number of scattered waves of random phases to produce speckle pattern. This leads to decrease the spatial autocorrelation between the speckle patterns. These explanations confirm the experimental and theoretical results presented in Figures 1-3 and Figure 9 \& Figure 10. While, the effect of the surface roughness on the spatial normalized autocorrelation function is not pronounced. The behavior of the spatial autocorrelation function for circular and square apertures is in good agreement with results already reported in literature [13] [14] [15] [17]. 


\section{Conflicts of Interest}

The authors declare no conflicts of interest regarding the publication of this paper.

\section{References}

[1] Goodman, J.W. (1975) Statistical Properties of Laser Speckle Patterns. In: Dainty, J.C., Ed., Laser Speckle and Related Phenomena, Springer Verlag, Berlin, 9-74. https://doi.org/10.1007/BFb0111436

[2] Moustafa, N.A., El-Nicklawy, M.M., Hassan, A.F. and Ibrahim, A.K. (2013) Effect of Partially Coherent Light on the Contrast of Speckle Patterns Obtained Using Digital Image Processing of Speckle Photography. Optics and Photonics Journal, 3, 324-329. https://doi.org/10.4236/opj.2013.35050

[3] El-Nicklawy, M.M., Abd-Rabou, A.M. and Elmahdy, A.E. (2018) New Analytical Expression for Speckle Contrast of Spectral Light Broadening. Journal of Optics, 20, Article ID: 015602. https://doi.org/10.1088/2040-8986/aa9a1d

[4] Ruffing, B. (1986) Application of Speckle-Correlation Methods to Surface-Roughness Measurement: A Theoretical Study. Journal of the Optical Society of America A, 3, 1297-304. https://doi.org/10.1364/JOSAA.3.001297

[5] Leger, D. and Perrin, J.C. (1976) Real-Time Measurement of Surface Roughness by Correlation of Speckle Patterns. Journal of the Optical Society of America, 66, 1210-1217. https://doi.org/10.1364/JOSA.66.001210

[6] Ruffing, B. and Fleischer, J. (1985) Spectral Correlation of Partially or Fully Developed Speckle Patterns Generated by Rough Surfaces. Journal of the Optical Society of America A, 2, 1637-1643. https://doi.org/10.1364/JOSAA.2.001637

[7] Léger, D., Mathieu, E. and Perrin, J.C. (1975) Optical Surface Roughness Determination Using Speckle Correlation Technique. Applied Optics, 14, 872-877. https://doi.org/10.1364/AO.14.000872

[8] Ohtsubo, J. and Asakura, T. (1976) Statistical Properties of Speckle Patterns Produced by Coherent Light at the Image and Defocus Planes. Optik, 45, 65-72.

[9] Xiang, J.N., Wu, Z.S., Hua, X.X. and Wang, M.J. (2013) Speckle Statistical Properties of Gaussian Beam from a Semi-Rough Target in the Atmospheric Turbulence. Optik, 124, 6760-6764. https://doi.org/10.1016/j.ijleo.2013.05.081

[10] Wang, Q. (2011) Theoretical Study on the Statistical Properties of Phase Difference between Two Interfering Speckle Fields. Optics Communications, 284, 5233-5239. https://doi.org/10.1016/j.optcom.2011.07.014

[11] Hinsch, K.D., Fricke-Begemann, T., Gülker, G. and Wolff, K. (2000) Speckle Correlation for the Analysis of Random Processes at Rough Surfaces. Optics and Lasers in Engineering, 33, 87-105. https://doi.org/10.1016/S0143-8166(00)00035-X

[12] Freud, I. and Kessler, D.A. (2008) Singularities in Speckled Speckle: Statistics. Optics Communications, 281, 5954-5967. https://doi.org/10.1016/j.optcom.2008.09.029

[13] Mysina, N.Y., Maksimova, L.A., Gorbatenko, B.B. and Ryabukho, V.P. (2015) Spatial Correlation and Probability Density Function of the Phase Difference in a Developed Speckle-Field: Numerical and Natural Experiments. Quentum Electronics, 45, 979-988. https://doi.org/10.1070/QE2015v045n10ABEH015560

[14] Uno, K., Uozumi, J. and Asakura, T. (1995) Speckle Clustering in Diffraction Patterns of Random Objects under Ring-Slit Illumination. Optics Communications, 114, 203-210. https://doi.org/10.1016/0030-4018(94)00653-C 
[15] Ibrahim, M., Uozumi, J. and Asakura, T. (1997) On the Generation of Clustered Speckles Due to Ring-Slit Illumination. Optik, 106, 33-41.

[16] Maack, T., Kowarschik, R. and Notni, G. (1998) Effect of the Reference Beam in Speckle Interferometry. Optics Communications, 154, 137-144. https://doi.org/10.1016/S0030-4018(98)00238-7

[17] Ibrahim, M., Uozumi, J. and Asakur, T. (1998) Longitudinal Correlation Properties of Speckles Produced by Ring-Slit Illumination. Optical Review, 5, 129-137. https://doi.org/10.1007/s10043-998-0129-2 